\title{
Novel Alginate-Gelatin Hybrid Nanoparticle for Drug Delivery and Tissue Engineering Applications
}

\author{
Eun Mi Lee, ${ }^{1}$ Deepti Singh, ${ }^{2,3}$ Dolly Singh, ${ }^{2,3}$ Soon Mo Choi, ${ }^{2,3}$ \\ Sun Mi Zo, ${ }^{2,3}$ Seon Joo Park, ${ }^{1}$ and Sung Soo Han ${ }^{2,3}$ \\ ${ }^{1}$ Department of Life Sciences, Yeungnam University, Gyeongsan, Gyeongsangbuk-do 712-749, Republic of Korea \\ ${ }^{2}$ Biomaterials Lab, Department of Nano, Medical \& Polymer Materials, College of Engineering, Yeungnam University, \\ 280 Daehak-ko, Gyeongsan, Gyeongsangbuk-do 712-749, Republic of Korea \\ ${ }^{3}$ YU-ECI Medical Research Center, Yeungnam University, 280 Daehak-ko, Gyeongsan, Gyeongsangbuk-do 712-749, Republic of Korea
}

Correspondence should be addressed to Sung Soo Han; sshan@yu.ac.kr

Received 30 June 2014; Revised 1 October 2014; Accepted 26 October 2014; Published 16 November 2014

Academic Editor: Gaurav Mago

Copyright (C) 2014 Eun Mi Lee et al. This is an open access article distributed under the Creative Commons Attribution License, which permits unrestricted use, distribution, and reproduction in any medium, provided the original work is properly cited.

\begin{abstract}
Novel alginate-gelatin hybrid nanoparticles were fabricated using single oil in water $(\mathrm{O} / \mathrm{W})$ emulsification techniques. Physicochemical property of the particle was characterized using scanning electron microscopy and Fourier's transmission infrared spectroscopy. Particle size was determined using zeta potential metastasize analyzer and was found to be in range of 400-600 nm. AGNPs were used for culturing human keratinocytes for two weeks to check biocompatibility of synthesized AGNPs. 3-(4,5Dimethylthiazol-2-yl)-2,5-diphenyltetrazolium bromide (MTT) assay showed increased metabolic activity of cells cultured on AGNPs in comparison to two-dimensional (2D) system (control). Cellular attachment on nanoparticle was further confirmed using SEM and 4',6-diamidino-2-phenylindole staining. The drug release profile shows possible electrostatic bond between alginate and gelatin resulting in controlled release of drug from AGNPs. For the first time alginate-gelatin hybrid nanosystem has been fabricated and all results showed it can be used as potential system for delivery of drug and therapeutical agents to cells and can also be used for regenerative medicine applications.
\end{abstract}

\section{Introduction}

Nanosystem has potential power to enhance drug stability and with longer circulation time targeted delivery of therapeutical agent could be achieved [1-3]. One of the most important parameters in designing nanoparticle for in vitro and in vivo application is biocompatibility of this system [4]. Biocompatible nanosystem can be achieved by modulating different parameters such as fabrication techniques, polymers used for its synthesis, and types of cross-linking agents; however, among these, polymers play the most significant role as they not only enter framework that carries drug but also interact with biological fluid and cells in the in vivo system [5-8]. Using natural polymers such as alginate, chitosan, and gelatin has evoked great interest in the field of nanotechnology as these polymers exhibit all the above-mentioned properties and in addition they are also easily biodegradable [9-13].
In case of regenerative medicine, it combines the principles of drug delivery and tissue engineering by utilizing material and life science technology for generating tissue or organs with better biological activity and also uses these principles for fabricating a system for delivering bioactive and therapeutical compounds [14]. Alginate has been extensively used in this area as it naturally gives polysaccharide with anionic and hydrophobic characteristics. As an ideal polymer for scaffolding and as nanocarrier, it not only is biocompatible with appropriate microarchitecture but also provides appropriate mechanical strength and has good rate of degradation and ability to support residing cells for various biological functionality making alginate as most sort after polymer in biomedical application [15-20]. Furthermore, alginate chelating ability with divalent cations makes it simpler and easier to fabricate hydrogel or nanoparticle using alginate. Besides these advantageous characteristics alginate is also known to have $\mathrm{pH}$ dependent anionic nature and interacts well with 
proteoglycans and polyelectrolyte [19]. Due to its several advantages alginate has been broadly used for cell encapsulation, DNA, proteins, vaccines, and drug delivery especially for oral delivery of insulin [20].

Similarly, gelatin is most versatile naturally occurring polymer widely used in regenerative medicine and pharmaceutical company. Gelatin is known for its exceptional properties such as being biocompatible and biodegradable with potential of different chemical modification obtained by partial hydrolysis of collagen [21-23]. It is the polyampholyte with both anionic and cationic along with hydrophobic group found in approximate ratio of 1:1:1 that makes this polypeptide special. Gelatin polymer chain is $\sim 12 \%$ negatively charged due to presence of glutamic and aspartic acid, 13\% positively charged due to presence of arginine and lysine, and $\sim 11 \%$ of its chain hydrophobic ascribed to presence of isoleucine, methionine, leucine, and valine whereas proline, glycine, and hydroxyproline constitute the rest of the polymer chain $[24,25]$. Gelatin based nanoparticles have been fabricated using different techniques such as desolation which uses an agent (acetone or alcohol) for dissolving gelatin in aqueous solution; this is done for dehydrating polymer chain that results in conformational change (stretched to coil) [26]. Another simple method of fabricating gelatin nanoparticle (NP) is reverse phase microemulsion, in which aqueous solution of gelatin is further mixed in surfactant containing solution (SDS or SBES in $n$-hexane) followed by cross-linking [27]. No matter which technique is used for fabrication, gelatin nanoparticle (GNP) has been extensively utilized for delivery of both hydrophobic and hydrophilic anticancer drugs, proteins, vaccine, and gene delivery [26, 27].

Even though both of these polymers have been found to have potential uses in regenerative medicine and are known for their advantageous characteristics, this is the first time alginate-gelatin nanoparticle (AGNP) is reported. In this study, we have synthesized alginate-gelatin nanoparticles as scaffold and drug delivery system. To further demonstrate their potential application in regenerative medicine, we have cultured human keratinocytes and checked for the biocompatibility and suitability of the nanosystem for cellular growth.

\section{Materials and Methods}

2.1. Chemicals Used for Experiments. Alginate (sodium salt), gelatin (from porcine skin, MW 60,000), glutaraldehyde $(25 \% \mathrm{v} / \mathrm{v})$, phosphate buffer saline (PBS), Dulbecco's modified Eagle's medium (DMEM), penicillin, streptomycin, dimethyl sulphoxide (DMSO), fetal bovine serum (FBS), (4,5-dimethylthiazol-2-yl)-2,5-diphenyltetrazolium bromide (MTT) were all purchased from Sigma Chemical Co., (St. Louis, MO, USA). Live/Dead staining kit was obtained from Life Technology-Invitrogen, USA. Human keratinocytes were purchased from Korean Cell Line Bank, Seoul, Republic of Korea. Grape seed oil (100\% purity) was obtained from Dong Yang Science, Daegu, Republic of Korea.

2.2. Alginate-Gelatin Nanoparticle (AGNP) Fabrication. Alginate $(250 \mathrm{mg})$ and gelatin $(500 \mathrm{mg})$ were prepared using oil/water emulsification techniques. Two polymers (alginategelatin) mixed thoroughly were added dropwise at $25^{\circ} \mathrm{C}$ in emulsification bath containing $150 \mathrm{~mL}$ grape seed oil (Dong Yang Science) and stirred speed was set at $650 \mathrm{rpm}$. Particles were allowed to form complexation for $30 \mathrm{~min}$ followed by recovering the particles by using cooled acetone $(60 \mathrm{~mL})$ for obtaining solid particles. Resultant particles were crosslinked for $4 \mathrm{~h}$ using $1 \%$ glutaraldehyde and later filtered and washed using double distilled water for several times to remove any traces of un-cross-linked aldehyde. Alginategelatin nanoparticle (AGNP) samples were stored in dry conditions for further analysis.

\subsection{Nanoparticle Characterization}

2.3.1. Size and Morphology. Morphology such as shape and occurrence of aggregation of AGNPs was studied using scanning electron microscopy (FEI Quanta 200) and bright field and confocal microscopy (Nikon fluorescent microscopy). For SEM imaging samples were vacuum-dried and platinumcoated using sputter coater. SEM was set up at high vacuum at $15 \mathrm{kV}$ with sample spot size fixed on $4.5 \mathrm{~mm}$ for imaging the particles. Nanoparticle size was assessed by Zetasizer and scattering particle size analyzer machine (Malvern Instrument, UK). Collected 8 random samples were studied by dispersing nanoparticles in oil at $25^{\circ} \mathrm{C}$ with detection angle set at $90^{\circ} \mathrm{C}$.

\subsubsection{Interaction of Polymers: Fourier's Transmission Infrared} Spectroscopy. Distribution of polymers in nanosystem and chemical bonding between alginate and gelatin were checked by Fourier's transmission infrared spectroscopy (FTIR) (PerkinElmer Spectrum 100, USA) which was used for obtaining infrared spectrum of AGNPs.

\subsubsection{Drug Release Profile Studies of Microparticle. AGNPs} were checked for drug release profile by recording the absorbance spectrum of doxorubicin (DOX) released from the nanosystem at $490 \mathrm{~nm}$ using UV (UV-2600, SHIMADZU) spectrophotometer [2]. DOX mixed in phosphate buffer saline (PBS, pH 7.4) was loaded on the AGNPs and incubated at $37^{\circ} \mathrm{C}$ with continuous stirring. PBS was also used as release media and $0.5 \mathrm{~mL}$ of PBS was taken out and absorbance was read using spectrophotometer at regular intervals for one week. All experiments were set up in quadruplets for concurrent readings and drug release rate is expressed in micromoles/mL for given time period calculated using standard curve plotted using different DOX concentration.

\subsection{Biocompatibility Testing of AGNPs}

2.4.1. In Vitro Cell Proliferation. AGNPs were tested for its biocompatible nature and ability to support cellular growth. Human keratinocytes cells with seeding density of $1 \times 10^{5}$ cells/ $\mathrm{mL}$ were cultured on ethanol sterilized AGNPs. Cells were briefly allowed to interact and settle onto nanoparticles before complete Dulbecco's modified Eagle's medium (DMEM) (with 10\% fetal bovine serum, 1\% penicillin/streptomycin) 


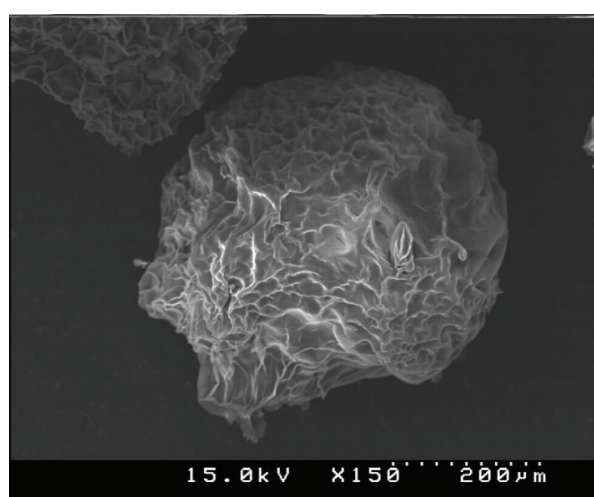

(a)

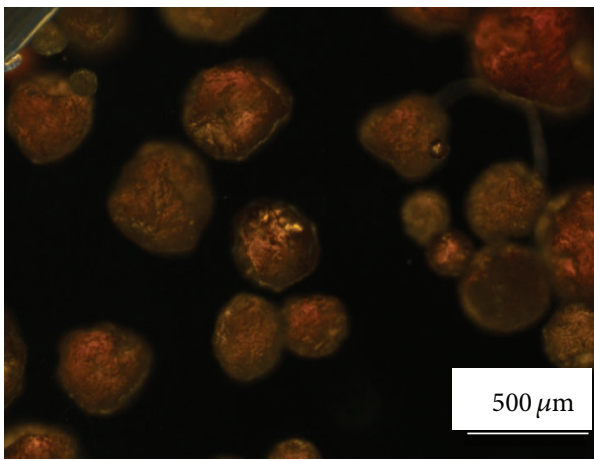

(c)

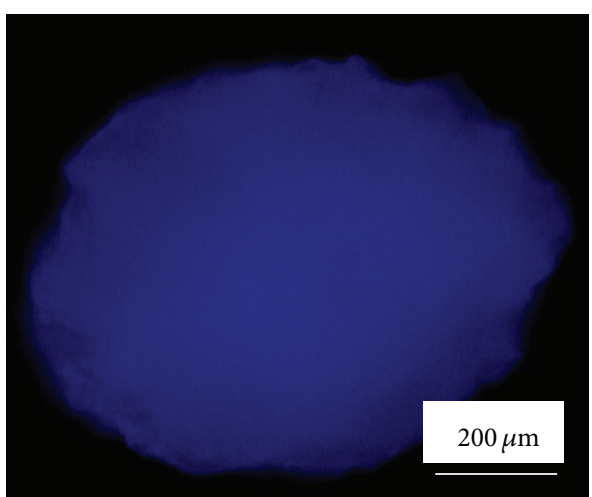

(b)

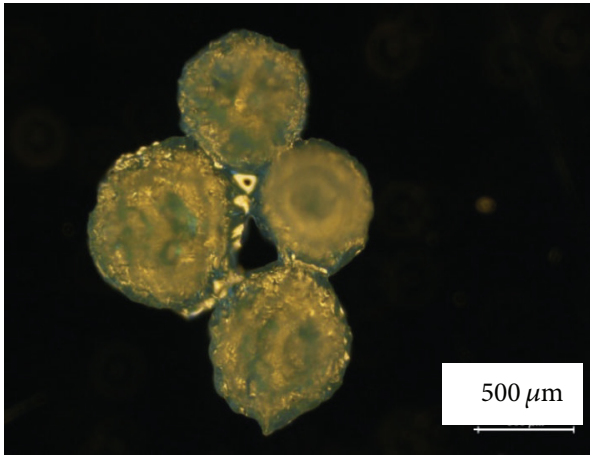

(d)

Figure 1: Microscopy images of AGNPs inSEM (a), UV-filter (b), dry state (c), and water swollen (d).

was added into each well and incubated at ideal cell culture conditions. Experiment was set up for two weeks and medium was regularly replaced with fresh medium and cell cultured on plates as $2 \mathrm{D}$ system was set as control with the same culture conditions as the experiment set.

2.4.2. Total Metabolic Activity of Cells. Metabolic activity of cells cultured as experiment and control was tested using standard 3-(4,5-dimethylthiazol-2-yl)-2,5-diphenyltetrazolium bromide (MTT) assay at every two-day interval. Briefly, medium is discarded from control and test samples followed by gentle washing of the wells with PBS (0.01 M, pH 7.4). MTT is widely regarded as an indirect technique for checking cellular growth and proliferation as mitochondrial enzyme found in metabolically active cells catalyzes MTT oxidation, which gives purple or dark violet color product which is dissolved in dimethyl sulphoxide (DMSO). Working solution of MTT ( $5 \mathrm{mg} / 1 \mathrm{~mL})$ was added to wells and incubated. After $3 \mathrm{~h}$ of incubation, MTT solution is discarded and DMSO was at a ratio $1: 3$ to every test well. Addition of DMSO results in dissolution of intracellular formazan crystals formed by MTT oxidization that develops into blue-violet color end product which is measured at $490 \mathrm{~nm}$ using UV-spectrophotometer (Shimdzu UV-2600) [24].

2.4.3. Imaging of Cells Proliferation on AGNPs. Cell seeded AGNPs were fixed by $2.5 \%$ glutaraldehyde for scanning electron microscopy analysis. 4',6-Diamidino-2-phenylindole
(DAPI) was directly added to AGNPs and 2D systems for fluorescent microscopic testing both of these microscopic techniques enable us to check for cellular attachment and growth and proliferation of cells to ANGPs.

2.4.4. Statistical Analysis. One-way variance test was performed using Sigma Stat for testing the significant difference between test and control. Significant difference was considered only when $P<0.03$. Experimental data are expressed as the means $\pm \mathrm{SD}$ and the in vitro and in vivo experiments were performed in set of three to obtain independent values.

\section{Results and Discussion}

3.1. Particle Size and Morphology. In the last few years many studies have been conducted on polysaccharide based 3D system for its potential use in drug delivery and tissue engineering $[2,23,27]$, however, with special efforts of fabricating nanosystem for delivering of anticancer agent to tumor site. Alginate is well-known biopolymer and polyelectrolyte regarded as being nonimmunogenic, nontoxic, and degradable. Similarly, gelatin has been exhaustively used for fabrication of nanosystems and scaffold due to its good solubility in water and the cell attracting moiety found along the polymer chain. Furthermore, gelatin presents the possibility of direct interaction with drug based on specific forces which can be achieved without chemically modifying gelatin [16, 17]. Despite the fact that both of these biopolymers have excellent 


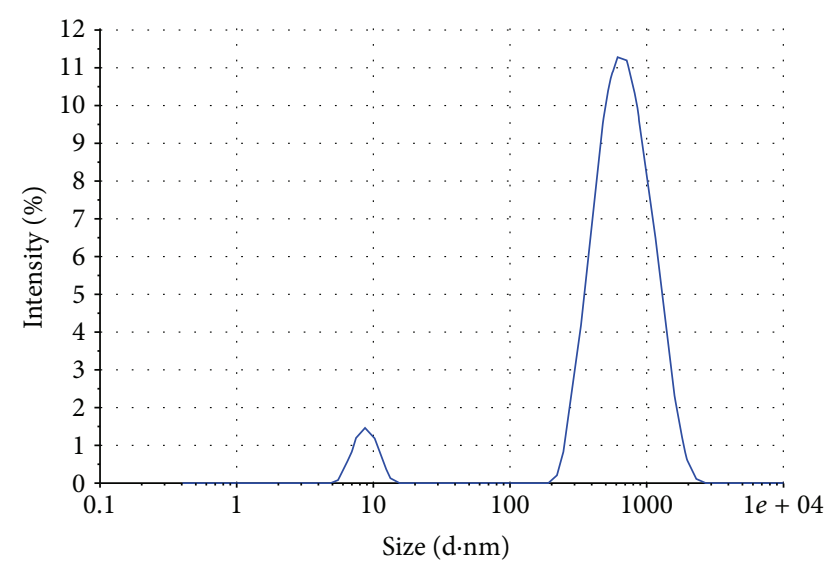

Figure 2: Zeta potential analyzer was used to measure the size distribution and average size of AGNPs was found to be in range of $489.5 \mathrm{~nm}$.

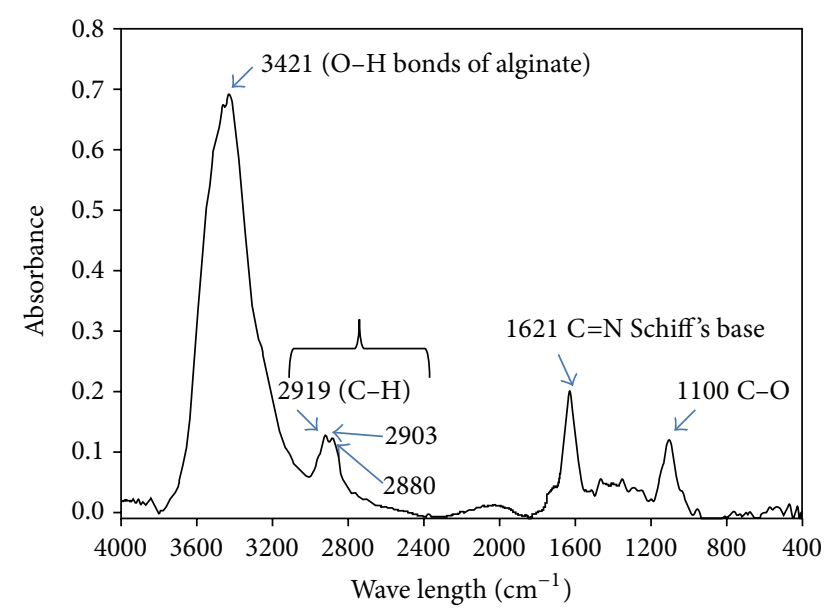

FIGURE 3: FT-IR spectrum showing the cross-linking between alginate and gelatin compared with individual polymer spectra.

properties, the combination of gelatin and alginate nanoparticle has not been so far reported.

AGNPs were prepared using standard single oil/water emulsification technique and cross-linked using glutaraldehyde after synthesis to stabilize the system. Different concentration of polymer was used for optimization; however, best polymer combination was of alginate with gelatin found to be of $1: 2(\mathrm{w} / \mathrm{w})$ ratios, respectively. Depending upon the polymer concentration, cross-linking percentage, stirring speed, and temperature, optimized size and shape of nanosystem could be achieved. It was found that higher complexation time resulted in unstable and extremely porous nanoparticle and lesser than 30 mins resulted in aggregated and nonhomogenous particle formation. Hence, it was found that each step was playing impetus role in the formation of homogenous nanosystem with higher reproducibility. SEM, fluorescent imaging (Figures 1(a)-1(d)), and zeta size analysis showed the average size of the nanosystem was in range of $400-600 \mathrm{~nm}$ (Figure 2) in which the smallest particle was found to be $100 \mathrm{~nm}$ and the largest was $800 \mathrm{~nm}$; however, on

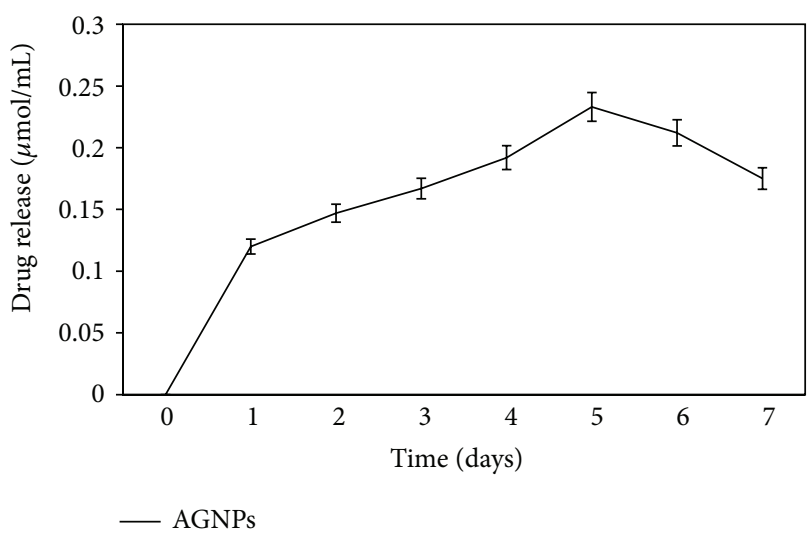

FIGURE 4: Release profile of DOX-loaded AGNPs shows controlled drug release for one week.

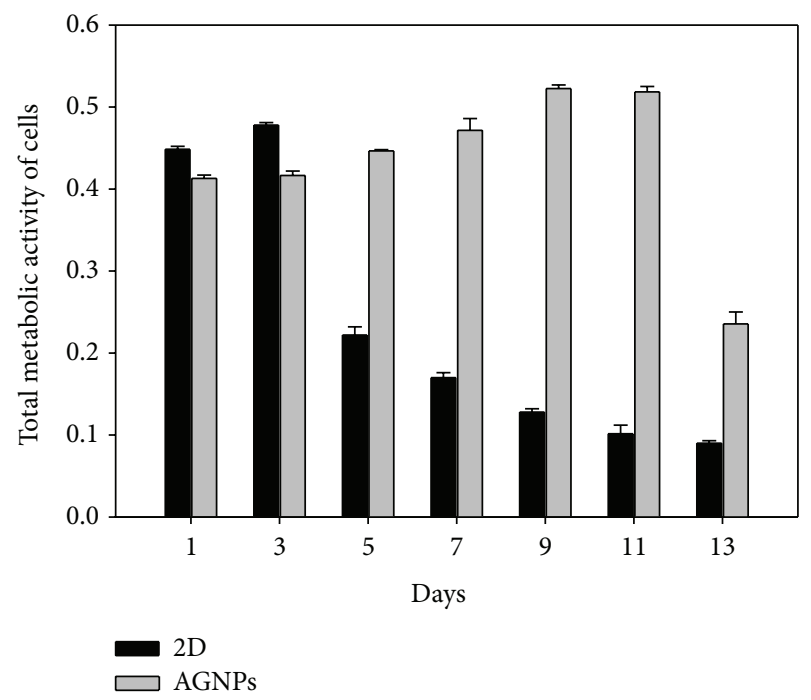

FIGURE 5: Human keratinocytes proliferation rate cultured on AGNPs analyzed using MTT and compared with 2D system (control).

an average it was $450 \pm(0.27) \mathrm{nm}$. No particle aggregation was observed during either cross-linking or drying when synthesized under constant stirring.

3.2. FT-IR Analysis for Chemical Bonding. FT-IR was used to determine the chemical bonding between alginate and gelatin. FT-IR of AGNPs reveals important absorption bands regarding ether, hydroxyl, and carboxylic functional groups. Stretching vibrations of alginate of $\mathrm{O}-\mathrm{H}$ bonds appear clearly in the range of $3000-3600 \mathrm{~cm}^{-1}$. Aliphatic C-H stretching vibrations were observed at $2919-2880 \mathrm{~cm}^{-1}$. Stretching vibration band at 1107 is attributed to the $\mathrm{C}-\mathrm{O}$ of pyranosyl ring of alginate and the $\mathrm{C}-\mathrm{O}$ stretching. FT-IR absorption bands at $1621 \mathrm{~cm}^{-1}$ are due to $\nu(\mathrm{C}=\mathrm{N})$ suggesting there is formation of Schiff's base along with complete absence of amide II (Figure 3). GA used as cross-linking agent is found 


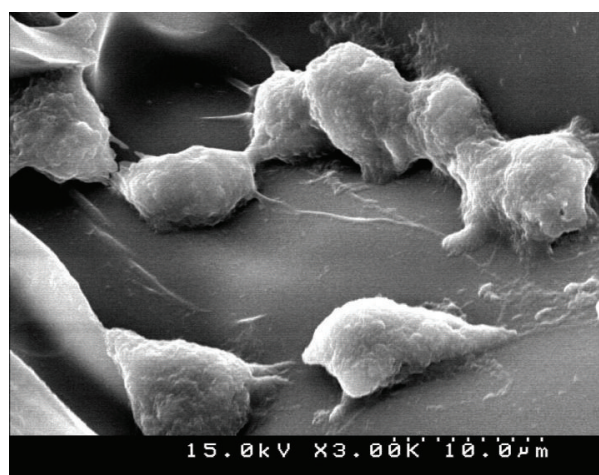

(a)

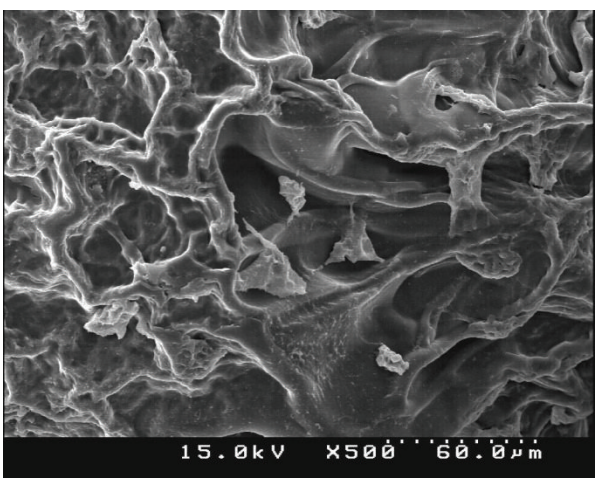

(c)

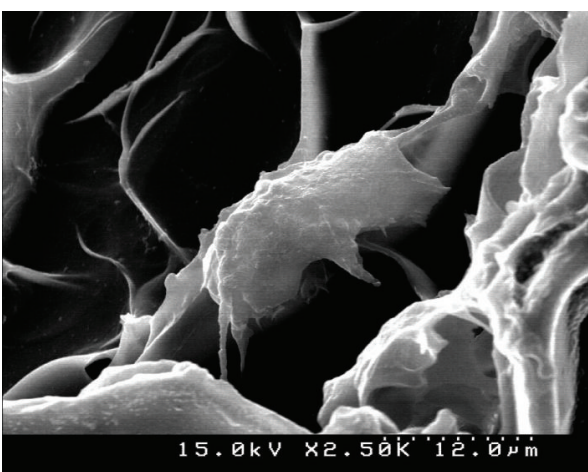

(b)

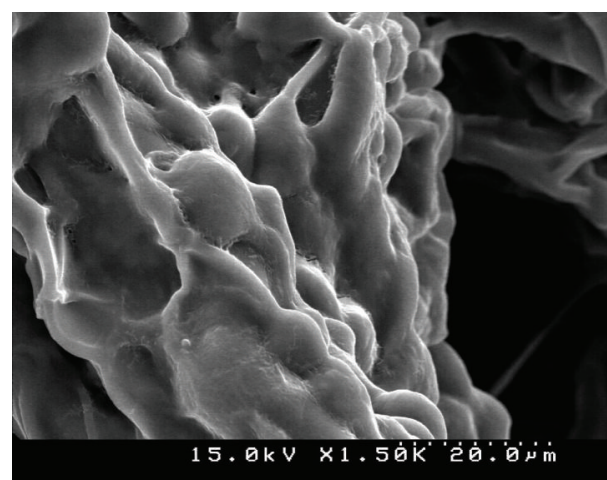

(d)

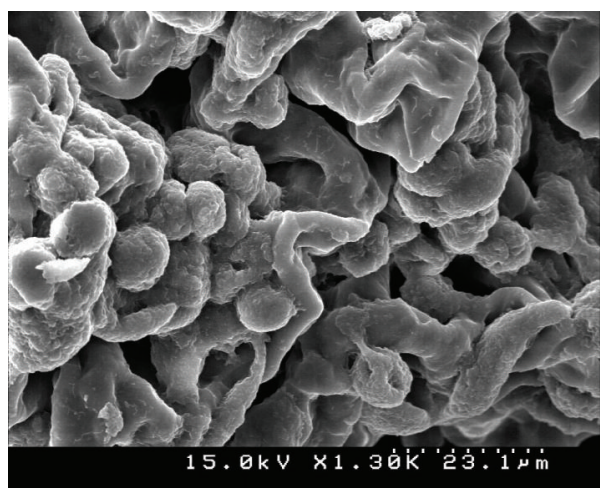

(e)

FIGURE 6: Scanning electron micrographs of alginate-gelatin nanoparticle (AGNP) seeded with human keratinocytes.

to be nonzero length cross-linker that induces bi- or polyfunctional cross-linking protein network structure by bridging free amino groups; hence, the amide II peek was not detected on FT-IR spectrum which would be seen in noncross-linked gelatin indicating involvement of its functional group with cross-linking of alginate.

3.3. Drug Release Profile of AGNPs. DOX is known potent drug with anticancer activity and has been extensively used for testing release properties of nano- and hydrogel materials intended to be used for therapeutical applications. For effective release of drug from nanosystem, the release medium needs to diffuse into the nanoparticle and dissolve loaded drug which is later released into surroundings, and both gelatin and alginate polymers are known for their hydrophilic property and hence movement of medium into particle and outside becomes easier. The drug release from AGNPs was examined by quantifying drug uptake and release in the media over period of incubation. Quantification of drug release from AGNPs system was obtained by plotting standard curve using different concentration of DOX (Figure 4). Standard graph helps in obtaining correlation equation which was further used for quantifying the concentration of drug released from AGNPs. From the 1st day DOX release was seen as steady and spectrophotometric study showed controlled and constant release of DOX over the period of 1 week. Drug uptake capacity was also quantified by allowing DOX absorbed. There have been different systems designed for attaining controlled drug release 

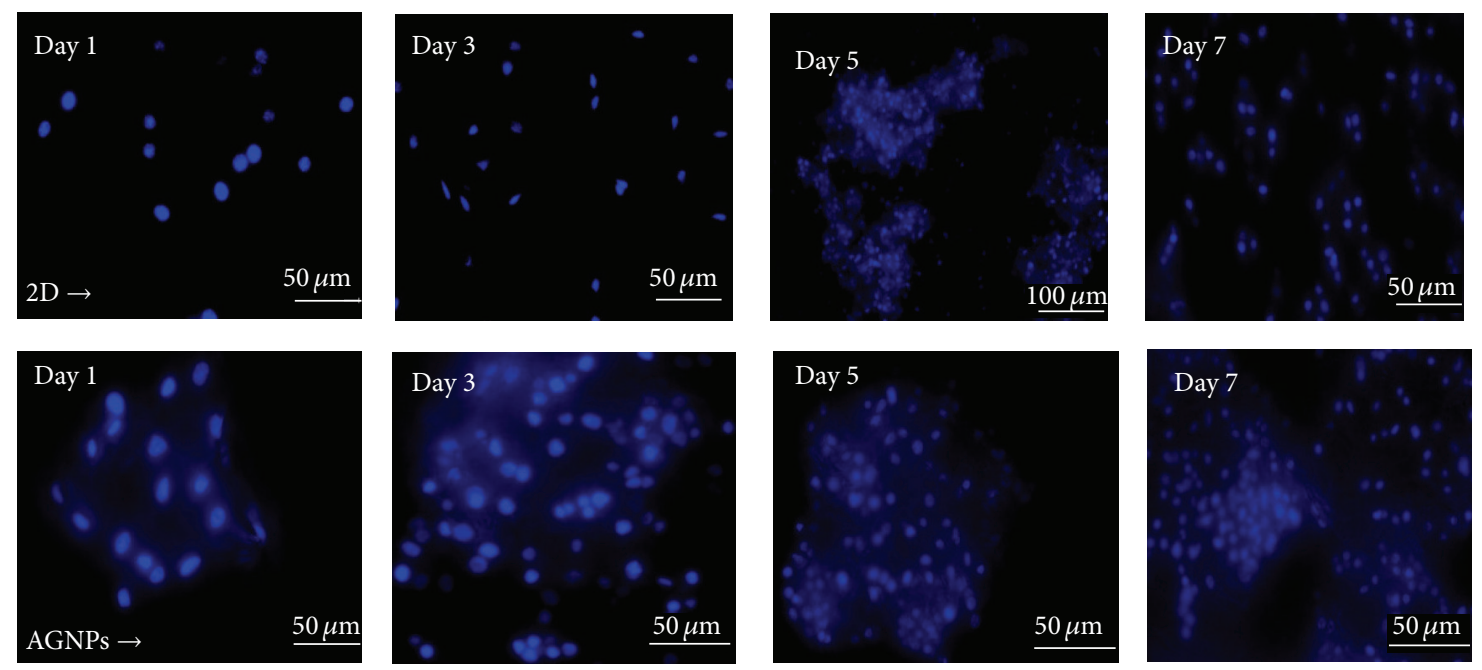

FIGURE 7: Nuclei staining of human keratinocytes on 2D (control) and alginate-gelatin nanoparticles (AGNPs).

which includes stimuli-responsive gels, hydrogel sheets, and different nanosystem; however, the biggest concern regarding these systems is its degradation properties. Using two natural polymers which are known to be biocompatible and biodegradable could help in overcoming the bottleneck faced during different therapeutical applications.

3.4. MTT Assay. Alginate and gelatin are both known biocompatible polymers; however, it is said that material that is compatible at macroscale can be toxic at nanoscale; hence, we investigated the effect of AGNPs on human keratinocytes. MTT assay was carried out to examine the biocompatible activity of AGNPs. Over the period of 2 weeks cell culture experiment showed initial higher cellular proliferation on 2D system (day 1); however, as days extended (day 5) cells cultured on AGNPs showed higher proliferation of human keratinocytes in comparison to control (2D). From the 7th day decrease in the metabolic activity of cells cultured in $2 \mathrm{D}$ is seen (Figure 5), whereas cells on AGNPs showed constant increasing trend till the end of the experiment. Even at different concentration of nanoparticles it was not found to have any cytotoxic effect of cells. Since the cells used were keratinocyte with doubling time of $48 \mathrm{hrs}$ and given that the space in 2D system is limited, the cells were found to be confluent and peel off from culture plate surface leading to lesser number of metabolically active cells. Cells in AGNPs show the maximum activity on the 9th day and are seen to maintain it till the 11th day; however, since the media was not changed throughout the experiment, it could have led to depletion in nutrients that in turn results in sharp decline in metabolic activity cells in both test and controls samples.

3.5. SEM and Imaging Analysis. To further confirm the results of MTT scanning electron microscopy was performed on AGNPs. SEM samples were examined on the same days as MTT (Figure 6) showed good cell attachment (day 1-A), proliferation (days 3 and 5-B and -C), and ECM production (day 7-D) on nanosystem. Cells were found attached on surface and inside the pore of the particles with high level of ECM production on AGNPs particles. Nuclei staining performed using DAPI (Figure 7) shows results similar to SEM with higher number of cells found at each interval of testing. In case of tissue engineering application nanosystem is used for achieving delivery of bioactive components and with precise architecture that facilitates cellular attachment, proliferation and tracking of cells in 3D construct could be extremely beneficial. Some requisites property for tissue engineered scaffold are sufficient pore size and homogenous distribution that can over period of time allow neo-vascularization formation; in addition, this should be able to attract cell for adhesion, proliferation, and matrix disposition. As particle for drug delivery and as matrix for tissue engineering, AGNPs are found to be potential candidate with ideal architecture to achieve sustained drug delivery, and matrix was also found to attract cellular adhesion and provide support for proliferation.

\section{Conclusion}

Alginate-gelatin nanoparticles can be the simplest delivery vehicle for any therapeutical agent since the drug release profile clearly shows controlled and steady release for over 2-week time. Along with controlled release the AGNPs were found to be highly biocompatible as the keratinocytes proliferation profile indicates that along with supporting cellular attachments AGNPs were found to support ECM production and cells were able to proliferate longer period of time. AGNPs can be potential system for both tissue engineering and regenerative medicine field.

\section{Conflict of Interests}

The authors declare that there is no conflict of interests regarding the publication of this paper. 


\section{Authors' Contribution}

Eun Mi Lee and Deepti Singh have contributed equally to this paper.

\section{Acknowledgment}

This research was fully funded by 2014 Yeungnam University Research Grant.

\section{References}

[1] A. O. Elzoghby, W. M. Samy, and N. A. Elgindy, "Protein-based nanocarriers as promising drug and gene delivery systems," Journal of Controlled Release, vol. 161, no. 1, pp. 38-49, 2012.

[2] D. Singh, D. Singh, S. M. Choi, and S. S. Han, "Enhanced proliferation and growth of human lung epithelial cells on gelatin microparticle loaded with Ephedra extracts," Journal of Nanomaterials, vol. 2013, Article ID 909120, 8 pages, 2013.

[3] J. J. Marty, R. C. Oppenheim, and P. Speiser, "Nanoparticles-a new colloidal drug delivery system," Pharmaceutica Acta Helvetiae, vol. 53, no. 1, pp. 17-23, 1978.

[4] J. A. Hubbell and A. Chilkoti, "Nanomaterials for drug delivery," Science, vol. 337, no. 6092, pp. 303-305, 2012.

[5] L. Jabr-Milane, L. van Vlerken, H. Devalapally et al., "Multifunctional nanocarriers for targeted delivery of drugs and genes," Journal of Controlled Release, vol. 130, no. 2, pp. 121-128, 2008.

[6] W. H. de Jong and P. J. A. Borm, "Drug delivery and nanoparticles: applications and hazards," International Journal of Nanomedicine, vol. 3, no. 2, pp. 133-149, 2008.

[7] A. Vonarbourg, C. Passirani, P. Saulnier, and J.-P. Benoit, "Parameters influencing the stealthiness of colloidal drug delivery systems," Biomaterials, vol. 27, no. 24, pp. 4356-4373, 2006.

[8] C. Chouly, D. Pouliquen, I. Lucet, J. J. Jeune, and P. Jallet, "Development of superparamagnetic nanoparticles for MRI: effect of particle size, charge and surface nature on biodistribution," Journal of Microencapsulation, vol. 13, no. 3, pp. 245-255, 1996.

[9] M. K. Gummerloch and E. A. Neuwalt, "Physiology and pharmacology of the blood-brain barrier," in Handbook of Experimental Pharmacology, M. W. B. Bradbury, Ed., vol. 103, pp. 525-542, Springer, Berlin, Germany, 1992.

[10] Y. Tabata, "Biomaterial technology for tissue engineering applications," Journal of the Royal Society Interface, vol. 6, no. 3, pp. S311-S324, 2009.

[11] A. Maruyama, T. Ishihara, N. Adachi, and T. Akaike, "Preparation of nanoparticles bearing high density carbohydrate chains using carbohydrate-carrying polymers as emulsifier," Biomaterials, vol. 15, no. 13, pp. 1035-1042, 1994.

[12] D. Singh, S. S. Han, and E. J. Shin, "Polysaccharides as nanocarriers for therapeutic applications," Journal of Biomedical Nanotechnology, vol. 10, no. 9, pp. 2149-2172, 2014.

[13] A. Cesàro, B. Bellich, and M. Borgogna, "Biophysical functionality in polysaccharides: From Lego-blocks to nano-particles," European Biophysics Journal, vol. 41, no. 4, pp. 379-395, 2012.

[14] R. Obregón, J. Ramón-Azcón, S. Ahadian et al., "The use of microtechnology and nanotechnology in fabricating vascularized tissues," Journal of Nanoscience and Nanotechnology, vol. 14, no. 1, pp. 487-500, 2014.

[15] J. S. Boateng, K. H. Matthews, H. N. E. Stevens, and G. M. Eccleston, "Wound healing dressings and drug delivery systems: a review," Journal of Pharmaceutical Sciences, vol. 97, no. 8, pp. 2892-2923, 2008.

[16] H. Zimmermann, S. G. Shirley, and U. Zimmermann, "Alginatebased encapsulation of cells: past, present, and future," Current Diabetes Reports, vol. 7, no. 4, pp. 314-320, 2007.

[17] I. D. Hay, Z. U. Rehman, A. Ghafoor, and B. H. A. Rehm, "Bacterial biosynthesis of alginates," Journal of Chemical Technology and Biotechnology, vol. 85, no. 6, pp. 752-759, 2010.

[18] U. Remminghorst and B. H. A. Rehm, "Bacterial alginates: from biosynthesis to applications," Biotechnology Letters, vol. 28, no. 21, pp. 1701-1712, 2006.

[19] D. Singh, D. Singh, S. M. ZO, and S. S. Han, "Nano- biomimetics for nano/micro tissue regeneration," Journal of Biomedical Nanotechnology, vol. 10, no. 10, pp. 3141-3161, 2014.

[20] S. N. Pawar and K. J. Edgar, "Alginate derivatization: a review of chemistry, properties and applications," Biomaterials, vol. 33, no. 11, pp. 3279-3305, 2012.

[21] P. Magadala and M. Amiji, "Epidermal growth factor receptortargeted gelatin-based engineered nanocarriers for DNA delivery and transfection in human pancreatic cancer cells," The AAPS Journal, vol. 10, no. 4, pp. 565-576, 2008.

[22] G. Kaul and M. Amiji, "Biodistribution and targeting potential of poly(ethylene glycol)-modified gelatin nanoparticles in subcutaneous murine tumor model," Journal of Drug Targeting, vol. 12, no. 9-10, pp. 585-591, 2004.

[23] A. O. Elzoghby, "Gelatin-based nanoparticles as drug and gene delivery systems: reviewing three decades of research," Journal of Controlled Release, vol. 172, no. 3, pp. 1075-1091, 2013.

[24] D. Singh, A. Tripathi, S. Zo, and S. S. Han, "Synthesis of composite gelatin-hyaluronic acid-alginate porous scaffold and evaluation for in vitro stem cell growth and in vivo tissue integration," Colloids and Surfaces B: Biointerfaces, vol. 116, no. 1, pp. 502-509, 2014.

[25] D. Singh, S. M. Zo, A. Kumar, and S. S. Han, "Engineering three-dimensional macroporous hydroxyethyl methacrylatealginate-gelatin cryogel for growth and proliferation of lung epithelial cells," Journal of Biomaterials Science, Polymer Edition, vol. 24, no. 11, pp. 1343-1359, 2013.

[26] S. Fuchs, J. Klier, A. May, G. Winter, C. Coester, and H. Gehlen, "Towards an inhalative in vivo application of immunomodulating gelatin nanoparticles in horse-related preformulation studies," Journal of Microencapsulation, vol. 29, no. 7, pp. 615625, 2012.

[27] K. Zwiorek, J. Kloeckner, E. Wagner, and C. Coester, "Gelatin nanoparticles as a new and simple gene delivery system," Journal of Pharmacy \& Pharmaceutical Sciences, vol. 7, no. 4, pp. 22-28, 2005. 

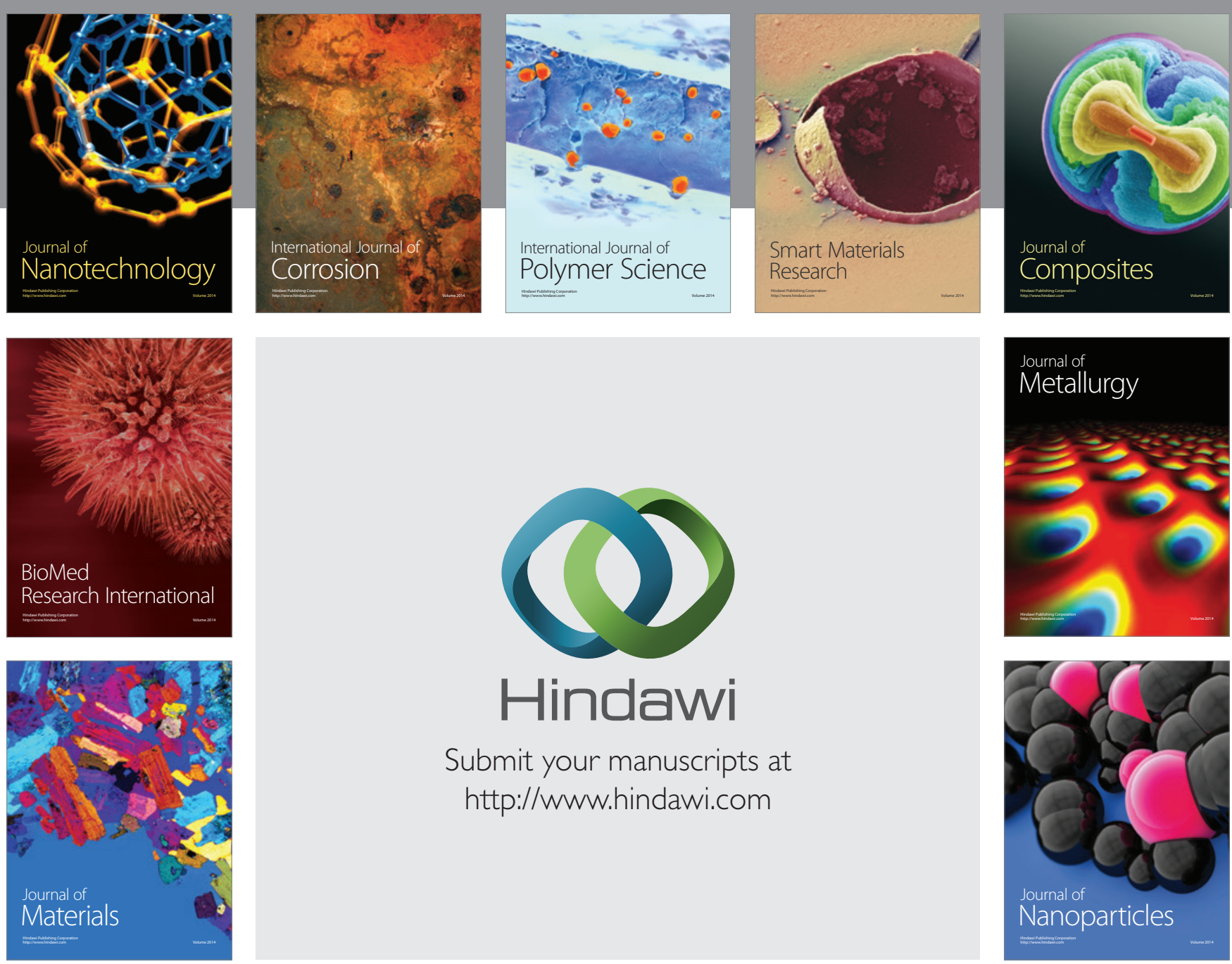

Submit your manuscripts at http://www.hindawi.com
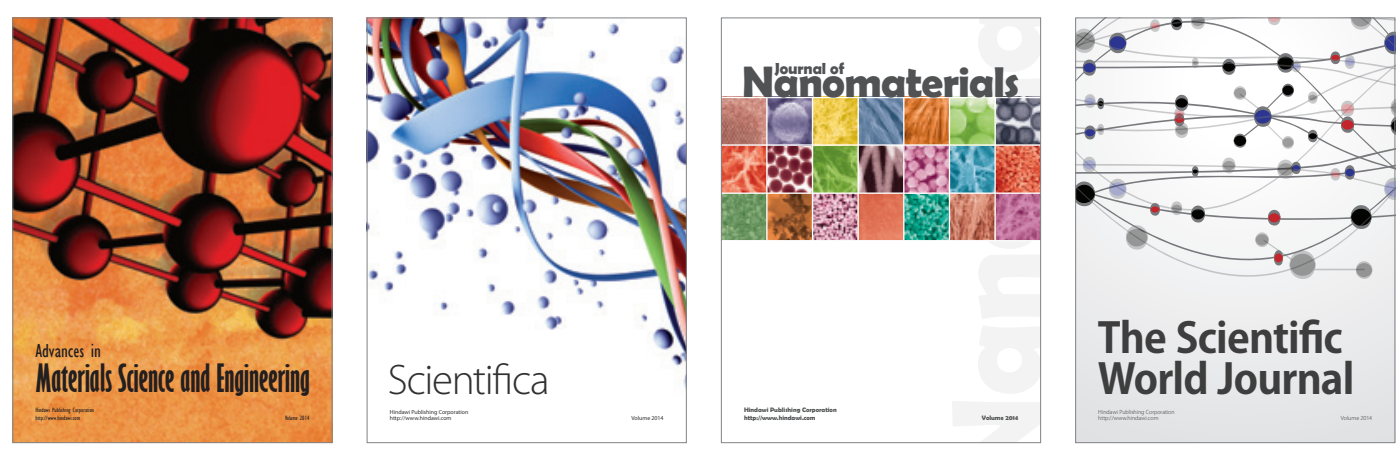

\section{The Scientific World Journal}
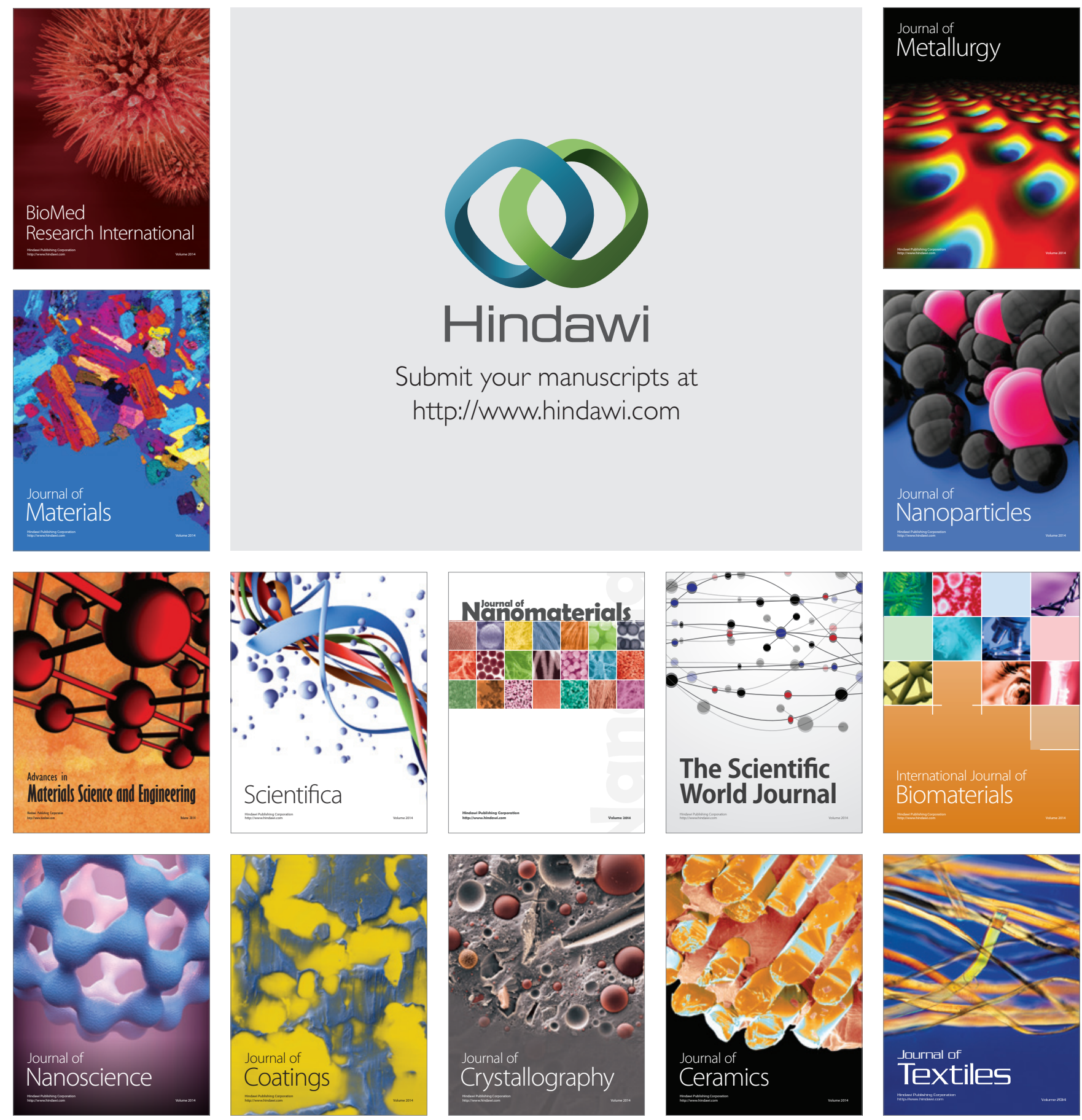